\title{
Forbidden island heights in stress-driven coherent Stranski-Krastanov growth
}

\author{
José Emilio Prietd* \\ Centro de Microanálisis de Materiales and Instituto Universitario "Nicolás Cabrera", \\ Universidad Autónoma de Madrid, E-28049 Madrid, Spain \\ Ivan Markov $\dagger$ \\ Institute of Physical Chemistry, Bulgarian Academy of Sciences, 1113 Sofia, Bulgaria
}

(Dated: February 4, 2020)

\begin{abstract}
The observed height distribution of clusters obtained in strained epitaxy has been often interpreted in terms of electronic effects. We show that some aspects can be explained classically by the interplay of strain and edge energies. We find that soft materials can transform directly from monolayer into thicker islands by two-dimensional (2D) multilayer nucleation and growth. There is a critical thickness decreasing with the force constant. Thinner islands are thermodynamically forbidden, due to the insufficient stress relaxation upon clustering particularly under tensile stress. At sufficiently large misfits the barrier for 2D multilayer nucleation is significantly smaller than the barrier for subsequent single-layer nucleation. The effects are found to be quantitatively reasonable and offer a plausible explanation for the absence of thin islands and $2 \mathrm{D}$ growth of flattop islands usually attributed to quantum size effects.

PACS numbers: 68.35.Md, 68.43.Hn, 68.55.Ac, 68.65.Hb
\end{abstract}

Nanostructures are very promising for optoelectronic and magnetic applications. For efficient operation, the shape, size and thickness distribution of small clusters are important parameters. Therefore it is crucial to understand the factors which control them. The epitaxy of metals on semiconductor surfaces at low temperatures $(130 \mathrm{~K}-180 \mathrm{~K})$ has been intensively studied in the last years [1, 2, 3, 4]. Some important observations are: (i) flat-top $\mathrm{Pb}$ islands with steep edges and a preferred height of 7 monolayers (ML) grow on the wetting layer on $\operatorname{Si}(111) 7 \times 7$ 1, 2, 3, 3], (ii) islands with thicknesses from 1 to 3 MLs are never observed [3, 6, 7]; (iii) flat-top $\mathrm{Pb}$ islands are preceded by pyramidal or dome-like clusters; clusters thinner than 3 MLs are never registered 8]; (iv) 2-ML thick flat-top $\mathrm{Ag}$ islands on $\mathrm{Si}(111)$ increase linearly in size with increasing coverage whereas ML islands preserve a nearly constant size of about $5 \mathrm{~nm}^{2}[8]$; (v) flat-top islands with a preferred height grow laterally without thickening [1, 7, 9]; (vi) Vertical growth of $\mathrm{Pb} / \mathrm{Si}(111)$ takes place by bilayer increments [7, 10].

The above observations were explained in terms of the energy lowering due to electron confinement and spilling of charge through the metal-semiconductor interface by Zhang, Niu and Shih, who coined for this reason the term "electronic growth" [11]. However, classical effects associated to strain relaxation are expected to contribute, as assumed to explain the fact that the increase of the aspect ratio of flat-top $\mathrm{Pb}$ islands is mediated by the formation and growth of strips (or rings) around the outer edges of the islands [12]. The aim of this paper is to show that some of the observations listed above, in particular (ii), (iii), (iv) and (v), can be explained classically in terms of the interplay of strain and edge energies.

Two mechanisms have been invoked to address the instability of planar growth against clustering. The first is the nucleationless development of instabilities of a certain wavelength that evolve into faceted three-dimensional (3D) islands [13]. The second is a nucleation behavior due to the competition of surface energy and strain relaxation [14]. Studies of the total energy per atom of islands of different heights as a function of their total number of atoms have shown that intermediate states with thicknesses increasing by ML steps are stable in separate consecutive intervals of volume [15, 16]. The rearrangement of mono- to bilayer islands was found to be a true nucleation process in compressed overlayers, in the sense that a small critical nucleus of the second layer is initially formed and then grows further up to the completion of the transformation. The associated energy displays a maximum at some number of atoms in the second level, which becomes very small for high enough values of the lattice misfit [17]. However, bilayer islands of expanded overlayers tend to require unrealistically high values of the lattice strain and of the island size to become stable against ML islands. Their transformation curves show an increase of the energy up to large second layer cluster sizes, making nucleation unlikely [17]. Also compressed overlayers of softer materials, or at lower misfits, are not expected to show nucleation behavior through this mechanism. We therefore set out to study the mono- to multilayer island transformation by using a simple atomistic model in $2+1$ dimensions.

We consider fcc $(100)$ crystallites with the shape of truncated square pyramids on a rigid layer of the same material with lattice constant $a$. In our model, atoms interact through a pair potential

$$
V(r)=V_{0}\left[\frac{\nu}{\mu-\nu} e^{-\mu(r-b)}-\frac{\mu}{\mu-\nu} e^{-\nu(r-b)}\right] .
$$


that in spite of its simplicity includes all necessary features to describe real materials (its strenght and anharmonicity are governed by the constants $\mu$ and $\nu$ [18]). The equilibrium atom separation is $b$, so that the lattice misfit is given by $\varepsilon=(b-a) / a$. We consider interactions only in the first coordination sphere. For the study of mono- to multilayer transformations, we assume model processes in which atoms are detached one by one from the edges of an initial square ML island, placed on top of it at the center and arranged in a $(X-1)$-ML thick structure as compact as possible up to the formation of a $X$-ML truncated pyramid. For every intermediate configuration, the cluster is allowed to relax by varying iteratively the atomic positions until all the forces fall below some negligible cutoff value. The energy change associated with the transformation at a particular stage is given by the difference between the energy of the incomplete multilayer island and that of the initial ML island.

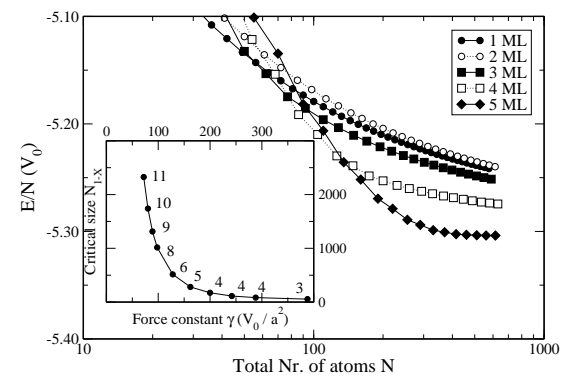

FIG. 1: Energy per atom of mono- and multilayer islands as a function of the total number of atoms. The misfit $\varepsilon$ amounts to $-7 \%$ and $\mu=2 \nu=26$. The insert shows the dependence of the critical size $N_{1-X}$ (beyond which 1-ML islands become unstable against $X$-ML islands) on the force constant $\gamma$. The threshold thickness $X$ is denoted by the numbers at each point.

Figure 1 shows the total energy per atom of expanded islands of different thicknesses as a function of the total number of atoms. It is seen that monolayer islands remain always stable against bilayer ones. At a critical size $N_{1-3}$, trilayer islands become stable and, with sizes increasing further, the ground state shifts to islands of thicknesses increasing in ML-steps. The insert of Fig. 1 illustrates the dependence of the critical size $N_{1-X}$ on the force constant $\gamma\left(\mu \nu V_{0}\right.$ in our model) for a fixed value of the misfit $(\varepsilon=-0.07)$. The threshold thickness below which multilayer islands are energetically unfavored steeply increases together with the critical volume with increasing softness (decreasing $\gamma$ ) of the epitaxial overlayer.

The stability regions of mono- and multilayer islands for both compressed and expanded overlayers are visualized in the phase diagram of Fig. 2, as a function of the number of atoms in the islands and the bulk strain energy per bond $\mathcal{E}=0.5 \gamma \varepsilon^{2} a^{2}$, which turns out to be the relevant scaling factor. As seen, ML islands are favoured

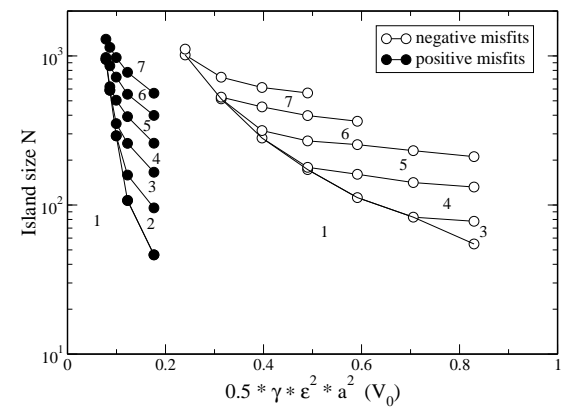

FIG. 2: Phase diagram showing the stability ranges of islands of different heights (given by the numbers in ML's) in coordinates of the total number of atoms and the bulk strain energy per bond $\mathcal{E}=0.5 \gamma \varepsilon^{2} a^{2}$, for positive and negative values of the misfit and for $\mu=2 \nu$ potentials.

at small number of atoms. For decreasing strain energies, the transition takes place for larger island sizes and to thicker islands. Expanded overlayers require larger strain energy to become unstable against clustering. Islands thinner than a certain number of layers are forbidden for energetic reasons. Result are shown for the particular class of potentials with $\mu=2 \nu$; other choices of $\mu$ and $\nu$ give points very close to those displayed. Different potentials (e.g. Lennard-Jones) are expected to give extremely similar results.

To compare with experiments, we estimate the strain energy per bond $\mathcal{E}$ from the force constant $\gamma$ of the overlayer material (in turn calculated as $\gamma=E b / 2\left(1-\nu_{P}\right.$ ), with $E$ the Young modulus and $\nu_{P}$ the Poisson ratio). Expressing $\mathcal{E}$ in units of the bond energy $V_{0}$ of the corresponding material, we get $0.15,3.08$ and 2.27 for the cases of $\mathrm{Pb}, \mathrm{Ag}$ and $\mathrm{Al}$ on $\mathrm{Si}(111)$, or $0.05,1.5$ and 1.3 $\mathrm{eV}$, respectively. Thus, a transition for $\mathrm{Pb} / \mathrm{Si}$ from $1 \mathrm{ML}$ to 8 or $9 \mathrm{ML}$ for islands of several thousands of atoms is expected, in reasonable agreement with experiments showing a preferred height of $7 \mathrm{ML}$ and scarce presence of thinner islands $[1,2,3,5,6,6,8]$. For $\mathrm{Ag}$ and $\mathrm{Al}$, the unrealistically high values of $\mathcal{E}$ (due to the failure of the harmonic approximation for such high values of the negative misfit) predict no absence of thin islands, as observed [8, 19] (see below). These estimations consider (100) coherent islands while most experiments are done on $\mathrm{Si}(111)$ and the islands are probably relaxed. However, they may retain approximate validity because the strain energy relaxation is partially balanced by the cost of disregistry. Furthermore, calculating similarly for the systems In and $\mathrm{Cd}$ on $\mathrm{Si}$ (to our knowledge not yet studied), we obtain 0.17 and $2.65 V_{0}$, respectively, thus predicting for In a similar behaviour as $\mathrm{Pb}$ (thin islands forbidden) in contrast to $\mathrm{Cd}$.

The results above point to a possible mechanism of 2D-3D transformation different from the consecutive formation of bilayer, trilayer, etc. islands [17, 20]: the direct nucleation of multilayer clusters whose thickness depends 
on the values of the force constant and the lattice misfit. We have studied this mechanism: Fig. 3 shows transformation curves from 1 to $3 \mathrm{ML}$ islands for two negative values of the misfit, -0.07 and -0.12 . Atoms are detached from the edges of the initial monolayer island and incorporated to the double steps of the bilayer island growing on top. The low-misfit curve is similar to the layer-bylayer curve shown in Fig. 5b of Ref. 17. The energy tends to increase all the way (the departure from monotonic behavior depends on the exact atomistic processes related to filling and depleting of atomic rows during the transformation process) and shows at the very end a sudden collapse due to the disappearance of the single and double steps to produce low-energy facets. On the contrary, the large-misfit curve shows in the beginning a nucleation behavior with a bilayer nucleus consisting of 22 atoms.

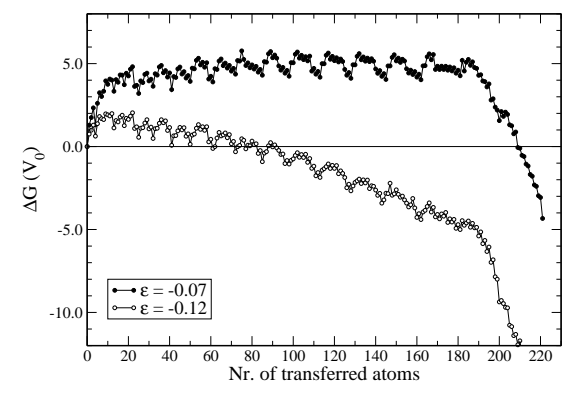

FIG. 3: Transformation curves representing the energy change in units of the bond energy $V_{0}$ as a function of the number of atoms transferred to the upper level for (a) $\varepsilon=-0.07$, and (b) $\varepsilon=-0.12$. The number of atoms in the initial monolayer island $(365 \approx 19 \times 19)$ gives a complete truncated trilayer pyramid $(12 \times 12+11 \times 11+10 \times 10)$ and $\mu=2 \nu=26$.

We consider first transformations from mono- to bilayer islands. Two processes accompany the island thickening: first, the distance $l$ between the upper and lower steps decreases. Since the step repulsion energy increases only as $l^{-2}$ [21], it is negligible except very close to the end of the tranformation. The second is the essential process, the increase of the total length of the steps, which in our model of square islands is given by $\Delta L=4\left(\sqrt{N_{0}-N_{2}}+\sqrt{N_{2}}-\sqrt{N_{0}}\right)$, with $N_{0}$ and $N_{2}$ being the number of atoms in the initial ML island and in the incomplete upper layer, repectively (note that, as a first approximation, there is no change in surface or interface energies, since there is no variation of the out-of-plane coordination of the atoms, and islands and wetting layer are composed of the same material). This process has three energetic contributions associated: the first is an increase in step energy, understood as the energetic cost of the unsaturated bonds, due to the reduced coordination at steps. The second is the relaxation of strain at steps and the third is the reduction of interlayer adhesion at steps due to the edge atoms climbing up in the potential valley created by their neighbors underneath. Only the strain relaxation favors the process of cluster- ing [17, 22]. The other two contributions oppose it, in addition to the step repulsion energy.

The sign of the misfit strongly affects the strain relaxation at steps. For both signs and for large enough islands, the bonds are almost completely relaxed at the edges and strained at the center. However, for intermediate values of island's size and misfit $(3-7 \%)$ 23, 24], the region with partially relaxed bonds extends considerably further in the case of compressed islands. Thus, as a result of anharmonicity, strain relaxation at steps is stronger for positive than for negative misfits of the same absolute value. The reduced coordination at steps, i.e. the number of unsaturated bonds, The number of edge atoms obviously does not depend on the sign of the misfit, while the reduced adhesion to out-of-plane neighbours is expected to depend weakly, because the interaction of an edge atom with its neighbours in the layer underneath necessarily involves attractive and repulsive forces in both cases. In compressed islands the strain relaxation at steps overcompensates the increase of the step energy (the total step length increases very fast in the beginning of the transformation). The balance turns for an island size which becomes very small for large enough values of the misfit. This results in nucleation-like behavior [17]. In expanded islands the weaker strain relaxation at steps requires larger values of the misfit to overcome the remaining contributions.

We consider next the instability of mono- against multilayer islands at small values of the force constant of the material. Owing to the weak strain relaxation at steps, the strain energy per atom decreases very weakly with thickness. A useful picture is to consider $n$-ML islands in coherent epitaxy as 1-ML islands but with $n$ times stronger bonds, i.e. $n$ times "stiffer" 25]. If the force constant of the material is small, the gain in strain energy upon the formation of ML islands on the first ML will be also small. Instead, bilayer islands will become energetically favoured with increasing number of atoms, i.e., the ground state will evolve from mono- to trilayer islands. These, being effectively "stiffer", relax the strain energy at steps more efficiently, overcoming the energetic cost of the bilayer step. For smaller values of the force constant tetralayers and even thicker islands will become stable against monolayer islands when the total size increases. This behavior is found also in compressed islands, shifted to smaller values of the force constant due to the anharmonicity of the interatomic potential.

For both compressed and expanded overlayers, the decrease of the strain energy per bond leads to an increase of the critical thickness $X$ at which the corresponding island becomes energetically stable against a ML island of the same size. A nucleation-like behavior is expected for this 1-X ML transformation. This basic result of our study, summarised in Fig. 2, implies that islands with insufficient thickness, i.e. with insufficient relaxation of strain energy at steps, will be forbidden to form for ther- 
modynamic reasons. This can explain the absence of $\mathrm{Pb}$ islands thinner than 4 ML [3, 6, 6, 6],

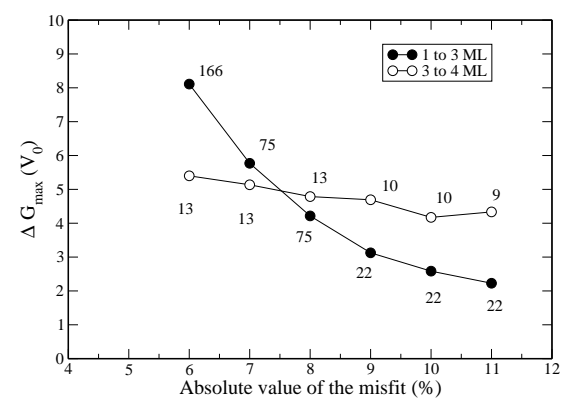

FIG. 4: Nucleation barriers in units of $V_{0}$ as a function of the absolute value of the negative misfit, for mono- to trilayer islands. The number of atoms in the critical nucleus is given at each point. Also shown is the barrier for monolayer nucleation on top of the trilayer island. The total number of atoms is 365 , and $\mu=2 \nu=26$.

Figure 4 shows a further important result. The monotonic decrease of the nucleation barrier height with misfit for the 1-3 ML transformation is characteristic of nucleation behaviour [17]. For small misfits, the barrier for the 3-4 ML transformation is smaller than for 1-3 ML, implying that, if 1-3 is possible, so will be also 3-4 or even higher. However, at larger absolute values of the misfit the barrier for formation of a bilayer nucleus on the first ML is significantly smaller than the barrier for formation of a ML nucleus on the trilayer, so that the growth of the 4th atomic level might be kinetically inhibited in this case. A similar intersection of the curves (not shown) is found for positive misfits, shifted to smaller absolute values. Thus, for large absolute values of the misfit the strain relaxation at the ML cluster growing on the trilayer island is not strong enough, overcoming the increase in step energy, to result in a small barrier height (this is essentially the same behavior as in the 1-2 ML transformation discussed above) Therefore, at sufficiently large misfits the growth in height can be strongly inhibited due to kinetics. For example, a factor 2 in the barrier height, as given by Fig 4 for high misfits reduces the nucleation rate several orders of magnitude at room temperature or below and 2D lateral growth will be preferred, as in fact observed [1, 7, 9]. In addition, the side walls act as better traps for adatoms than the flat top surface. The lateral growth by rearrangement of mono- to multilayer islands is strongly supported by the observation that 2ML thick flat-top Ag islands on $\mathrm{Si}(111)$ increase linearly in size with increasing coverage whereas ML islands preserve a nearly constant size of about $500 \AA^{2}$ [8], which can be interpreted as the critical size $N_{1-2}$. A clear evidence for a transformation process is the rearrangement of 2 to 3 -ML Fe islands on $\mathrm{Cu}_{3} \mathrm{Au}(001)$ upon annealing at $400 \mathrm{~K}$ [26, 27].

In conclusion, we find that materials with weak interatomic bonding will transform from ML islands di- rectly into thick islands by $2 \mathrm{D}$ multilayer nucleation and growth. Islands thinner than the critical thickness are thermodynamically forbidden due to the insufficient strain relaxation at steps, particularly when under tensile stress. At sufficiently large misfits the barrier for $2 \mathrm{D}$ multilayer nucleation is significantly smaller than the barrier for the subsequent single-layer nucleation. The above effects due to the interplay of step energy and strain relaxation are found to be quantitatively reasonable and offer a plausible explanation for the absence of thin islands and the 2D propagation of flat-top islands in epitaxial growth of metals on semiconductors.

This work was supported by projects MAT2005-03011 of the Spanish MEC and S-0505/MAT-0194 of the Comunidad de Madrid. J.E.P. also gratefully acknowledges financing by the programme "Ramón y Cajal" and fruitful discussions with Juan de la Figuera.

* Electronic address: joseemilio.prieto@uam.es

$\dagger$ Electronic address: imarkov@ipchp.ipc.bas.bg

[1] L. Gavioli et al. Phys. Rev. Lett. 82, 129 (1999).

[2] M. Hupalo et al. Phys. Rev. B 64, 155307 (2001).

[3] W.B. Su et al. Phys. Rev. Lett. 86, 5116 (2001).

[4] L. Floreano et al. Progr. Surf. Sci. 72, 135 (2003).

[5] V. Yeh et al. Phys. Rev. Lett. 85, 5158 (2000).

[6] S.H. Chang et al. Phys. Rev. B 65, 245401 (2002).

[7] M.M. Özer et al. Phys. Rev. B 72, 113409 (2005).

[8] W.B. Su et al. Phys. Rev. B 71, 073304 (2005).

[9] K. Budde et al. Phys. Rev. B 61, R10602 (2000).

[10] M. Hupalo et al. Surf. Sci. 493, 526 (2001).

[11] Z. Zhang, Q. Niu and C.-K. Shih, Phys. Rev. Lett. 80, 5381 (1998).

[12] H. Okamoto, D. Chen, and T. Yamada, Phys. Rev. Lett. 89, 256101 (2002).

[13] P. Sutter and M.G. Lagally, Phys. Rev. Lett. 84, 4637 (2000).

[14] J. Tersoff and F.K. LeGoues, Phys. Rev. Lett. 72, 3570 (1994).

[15] K.E. Khor and S. Das Sarma, Phys. Rev. B 62, 16657 (2000).

[16] J.E. Prieto and I. Markov, Phys. Rev. B 66, 073408 (2002).

[17] J.E. Prieto and I. Markov, Phys. Rev. B 72, 205412 (2005).

[18] I. Markov, Phys. Rev. B 48, 14016 (1993).

[19] H. Liu et al. Surf. Sci. 571, 5 (2004).

[20] S. Stoyanov and I. Markov, Surf. Sci. 116, 313 (1982).

[21] P. Nozières, in Solids far from Equilibrium, ed. by C. Godrèche, (Cambridge University Press, 1992), p. 1.

[22] P. Müller and R. Kern, Appl. Surf. Sci. 102, 6 (1996).

[23] I. Markov and A. Milchev, Surf. Sci. 145, 313 (1984).

[24] E. Korutcheva, A. M. Turiel and I. Markov, Phys. Rev. B 61, 16890 (2000).

[25] J.H. van der Merwe, J. Woltersdorf, W. A. Jesser, Mater. Sci. Eng. 81, 1 (1986).

[26] M. Canepa et al. Phys. Rev. B 62, 13121 (2000).

[27] A. Verdini et al. Phys. Rev. B 65, 233403 (2002). 